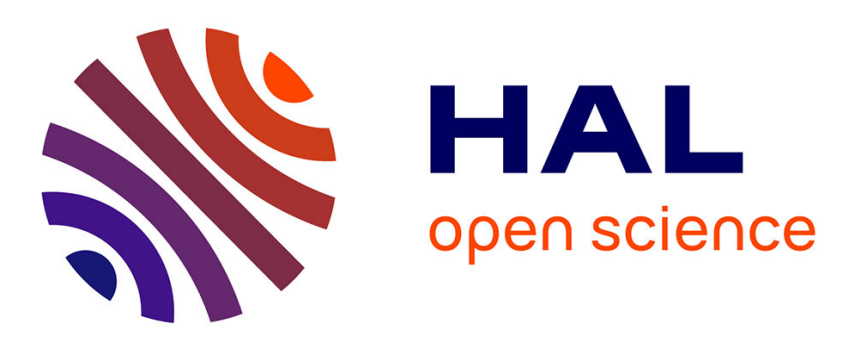

\title{
Generation of ultra-realistic synthetic echocardiographic sequences
}

\author{
Mathieu de Craene, Martino Alessandrini, Pascal Allain, Stephanie \\ Marchesseau, I Waechter-Stehle, J Weese, E Saloux, H. G. Morales, R \\ Cuingnet, Hervé Delingette, et al.
}

\section{To cite this version:}

Mathieu de Craene, Martino Alessandrini, Pascal Allain, Stephanie Marchesseau, I Waechter-Stehle, et al.. Generation of ultra-realistic synthetic echocardiographic sequences. Biomedical Imaging (ISBI), 2014 IEEE 11th International Symposium on, Apr 2014, Beijing, China. pp.73 - 76, 10.1109/ISBI.2014.6867812 . hal-01095133

\section{HAL Id: hal-01095133 \\ https://hal.inria.fr/hal-01095133}

Submitted on 15 Dec 2014

HAL is a multi-disciplinary open access archive for the deposit and dissemination of scientific research documents, whether they are published or not. The documents may come from teaching and research institutions in France or abroad, or from public or private research centers.
L'archive ouverte pluridisciplinaire HAL, est destinée au dépôt et à la diffusion de documents scientifiques de niveau recherche, publiés ou non, émanant des établissements d'enseignement et de recherche français ou étrangers, des laboratoires publics ou privés. 


\title{
GENERATION OF ULTRA-REALISTIC SYNTHETIC ECHOCARDIOGRAPHIC SEQUENCES
}

\author{
M. De Craene ${ }^{*}$, M. Alessandrini ${ }^{\star}$, P. Allain*, S. Marchesseau ${ }^{\wedge}$, I. Waechter-Stehle ${ }^{\vee}, J$. Weese ${ }^{\vee}$,

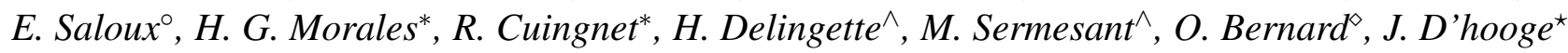 \\ * Medisys, Philips Research Paris, ${ }^{\star}$ Cardiovascular Imaging and Dynamics, KU Leuven, \\ ${ }^{\circ}$ Department of cardiology, CHU Caen, ${ }^{\vee}$ Philips Research Hamburg, \\ ${ }^{\wedge}$ Asclepios Project, Inria, ${ }^{\triangleright}$ CREATIS, CNRS UMR5220, Inserm U1044, INSA-Lyon, Université Lyon 1.
}

\begin{abstract}
This paper proposes a new simulation framework for generating realistic 3D ultrasound synthetic images that can serve for validating strain quantification algorithms. Our approach extends previous work and combines a real ultrasound sequence with synthetic biomechanical and ultrasound models. It provides images that fairly represent all typical ultrasound artifacts. Ground truth motion fields are unbiased to any tracking algorithm and model both healthy and pathological conditions.
\end{abstract}

Index Terms - Modeling of image formation; Ultrasound imaging; Motion detection and tracking.

\section{INTRODUCTION}

Quantification of cardiac motion and deformation/strain from 3D cardiac Ultrasound (3D US) is an active area of research. Despite encouraging preliminary studies, a widespread dissemination of these tools in clinical diagnostics is held back because no solid quality assurance methodology exists [1].

The validation of strain imaging methods has been addressed in several ways [1]: in vivo in human subjects by using tagged magnetic resonance (tMRI) as reference method; in vivo in open-thorax animal preparations by using sonomicrometry as a reference; in vitro by using a physical cardiac phantoms and sonomicrometry as reference and in silico using fully synthetic sequences [2, 3, 4].

In particular this latter solution presents the following advantages: it does not require inter-modal registration (unlike tMRI), and it provides the reference motion in every point of the heart (unlike sonomicrometry). Unfortunately, the level of realism of existing simulation techniques is highly unsatisfactory: surrounding structures such as papillary muscles and heart valves are typically neglected as well as typical artifacts such as reverberations, clutter noise, signal dropout and local intensity variations due to changing cardiac fiber orientation (see Fig. 1).

For this reason, recently two new simulations approaches

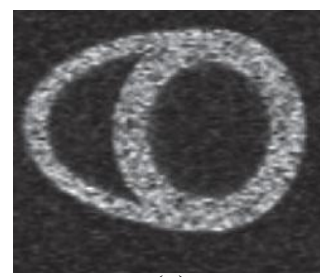

(a)

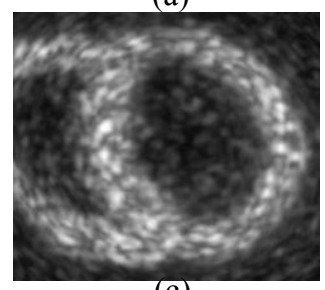

(c)

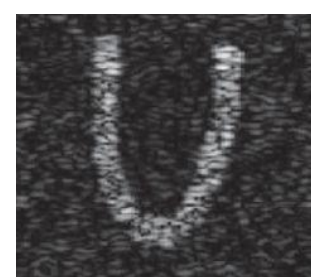

(b)

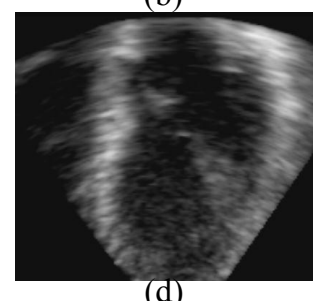

(d)
Fig. 1. Synthetic echocardiographic images used in [2](a), [3](b), [4](c) and using the framework proposed in this paper (d).

were proposed in which a clinical ultrasound recording was used as an intensity template for the simulated data set [5, $6]$. However, both solutions suffer from intrinsic limitations. In [5] the simulated cardiac motion is the result of a motion estimator applied to the template sequence. As a consequence no control on the simulated motion is possible. Also, errors of the adopted estimator reflect directly on the simulated motion. Finally, a bias can be expected in the comparison of different algorithms towards estimators of the same class as the one used to determine the synthetic motion.

On the other side, in [6] the synthetic sequence is obtained by warping directly the grayscale images of the template acquisition according to the desired motion field and this unavoidably introduces unrealistic warping artifacts in the resulting speckle texture.

In this paper we present a framework for the generation of $2 \mathrm{D}$ or $3 \mathrm{D}$ ultra-realistic synthetic echocardiographic sequences overcoming the limitations of existing approaches. Left and right ventricular anatomies are obtained through model-based segmentation of a real clinical recording. A synthetic motion field is then obtained by an electromechani- 

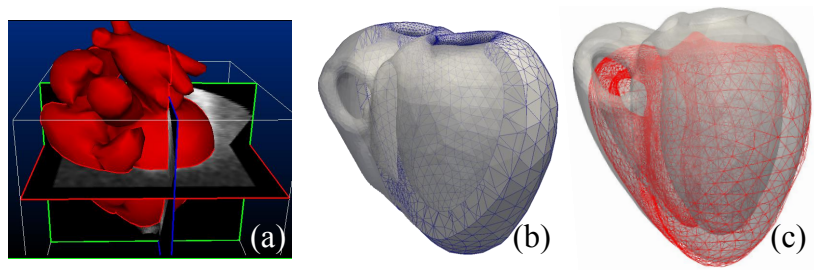

Fig. 2. From a 3D US image to a mechanical simulation result: (a) an average shape model is deformed to match intensity profiles in the image with a training database [9]; (b) from the resulting surface mesh, a volumetric tetrahedral mesh is generated, (c) a sequence of meshes is generated through the SOFA framework [7], here showing only the overlay between end-diastolic (gray) and end-systolic meshes (red).

cal model (E/M) [7]. The E/M model provides the reference for heart motion and derived quantities and can generate a continuous range of pathological patterns by progressively altering the value of contraction and/or conduction parameters. As in [5] a real clinical recording is adopted as template for local tissue echogenicity, which allows simulating surrounding structures as well as US artifacts. Finally, an US simulation environment is used to model the image formation [8], allowing full control over the transducer's parameters and ensuring realistic speckle patterns.

\section{PROPOSED SIMULATION FRAMEWORK}

In our framework, the generation of the synthetic ultrasound image sequence requires to define at every frame a set of discrete scattering sites and their respective echogenicity values. The E/M simulation affects the coordinates of scatterers inside the myocardium. Echogenicity values are obtained from the intensity of a real clinical recording. Integrating the mechanical simulation result with an ultrasound image sequence was performed in this paper by combining two elements. First, a patient-specific volumetric mesh is constructed by segmenting the first image frame (see Sect. 2.1). All E/M simulations are then performed on this geometry. Second, the echogenicity of scatterers moving in the simulation space is computed from the image intensities. This requires to compute a set of transformations that relate a coordinate from the simulation space to the image (see Sect. 2.2). The combination of this set of transformations with the mechanical simulation result is described in Sect. 2.3.

\subsection{D image segmentation, mesh generation and me- chanical simulation}

Left and right ventricular geometries are first extracted from the first frame of a template 3D US image sequence (i.e. a real clinical recording). Hereto, the four chambers were automatically extracted from the 3D image using a model-based segmentation technique initially developed for CT images [9] and recently extended to ultrasound.

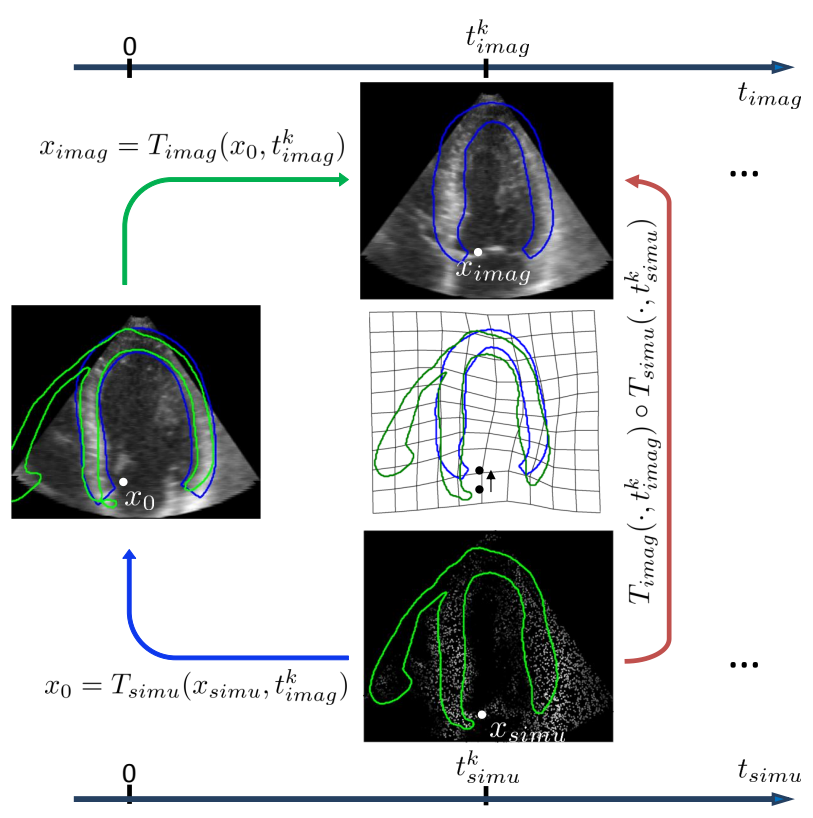

Fig. 3. Spatiotemporal mappings used to estimate the echogenicity of a background scatterer, from simulation to image spaces.

From the segmentation result on the first image frame, two outputs are further produced. The first output is a dynamic sequence of LV endo- and epicardial segmentations produced by tracking and manual editing. The editing is done in $4 \mathrm{D}$ to ensure a smooth propagation of manual updates. The result is a set of landmarks over time sampling the LV endoand epicardial surfaces regularly in the circumferential and longitudinal directions. This processing is part of the QLab software (3DQ Advanced plugin) [10].

The second output is a volumetric tetrahedral mesh obtained by applying the NetGen (http://www.hpfem. jku.at/netgen/) software to the surface mesh. Prior to mesh generation, the right ventricle was first extruded along the mesh normals to produce a RV epicardial surface. We then applied the simulation technique described in [7] and implemented in the SOFA framework (http: //www.sofa-framework.org/) for generating two simulations of healthy and ischemic cases. The ischemic case is produced by altering the contractility of segments perfused by the left circumflex artery [4].

\subsection{Spatial and temporal matching between image and simulation sequences}

The image and simulation sequences have different dynamics that have to be matched. A transformation between image and simulation times is computed by linear interpolation between temporal landmarks defined on both sequences. For each simulation frame indexed by $t_{\text {simu }}^{k}$, we compute $t_{\text {imag }}^{k}$ being the interpolated time in the real sequence. The closest image frames are defined as $t_{i m a g}^{k+}=\left\lceil t_{i m a g}^{k}\right\rceil$ and $t_{i m a g}^{k-}=\left\lfloor t_{i m a g}^{k}\right\rfloor$. From $t_{i m a g}^{k+}$ and $t_{i m a g}^{k-}$, two motion fields can then be de- 
rived for matching coordinates from the first image frame to these two times. These two mappings will be denoted as $T_{\text {imag }}\left(\cdot, t_{\text {imag }}^{k-}\right)$ and $T_{\text {imag }}\left(\cdot, t_{\text {imag }}^{k+}\right)$ where the first argument is a spatial coordinate in the domain of the first image frame. In our implementation, these mappings were computed from a Thin Plate Spline (TPS) transform parametrized by the 4D segmentation landmarks (Sect. 2.1).

In the simulation space, a set of transformations mapping each frame to the first frame are computed similarly as in the image space. They are noted as $T_{\text {simu }}\left(\cdot, t_{\text {simu }}^{k}\right)$ where the first argument is a spatial coordinate at frame $t_{\text {simu }}^{k}$. All mappings and coordinates notations are summarized in Fig. 3.

\subsection{Scatter map generation}

The first step is to generate a scatter map on the first frame. The scatter map contains the coordinates and echogenicity of each scatterer. As the E/M mesh matches the first image frame, the mesh can be used to differentiate foreground (within myocardium) from background scatterers. The scatterers coordinates on the first simulation frame are obtained by uniformly sampling $N$ points through the image domain. Parametric E/M mesh coordinates of foreground scatterers are stored for further use. Their echogenechogenicity is obtained by interpolating the intensity of the first frame of the template image sequence.

For generating the scatter map at time $t_{\text {simu }}^{k}>0$, we first move the $N_{M}$ scatterers within the myocardium by computing the absolute coordinates from the parametric coordinates computed on the first frame. We then redraw $N_{B}=N-N_{M}$ samples using an uniform distribution through the image domain, excluding the myocardial volume.

For each scatterer at coordinate $x_{\text {simu }}$ and time $t_{\text {simu }}^{k}$, we then define the position $x_{0}$ of that scatterer at time 0 . For foreground scatterers, $x_{0}$ is directly available since they are indexed consistently over time. For background scatterers, it can be computed as

$$
x_{0}=T_{\text {simu }}\left(x_{\text {simu }}, t_{\text {simu }}^{k}\right) .
$$

The echogenicity of every scatterer can then be defined as

$$
a=\left\{\begin{array}{cll}
(1-w) I_{t_{i m a g}^{k-}} \circ T_{i m a g}\left(x_{0}, t_{i m a g}^{k-}\right) & & \\
+w \cdot I_{t_{i m a g}^{k+}}^{k+} \circ T_{i m a g}\left(x_{0}, t_{i m a g}^{k+}\right) & \text { if } & x_{0} \notin \Omega_{M} \\
I_{0}\left(x_{0}\right) & \text { if } \quad x_{0} \in \Omega_{M}
\end{array}\right.
$$

where $t_{i m a g}^{k+}$ and $t_{i m a g}^{k-}$ are defined in Sect. 2.2, $\Omega_{M}$ stands for the myocardial domain at time 0 and $w$ is an interpolation weight defined as

$$
w=t_{\text {imag }}^{k}-t_{i m a g}^{k-} .
$$

Amplitudes computed from Eq. 2 are further calibrated using

$$
a^{\prime}=10^{\frac{K}{20}\left(\frac{a}{a_{M A X}}-1\right)}
$$
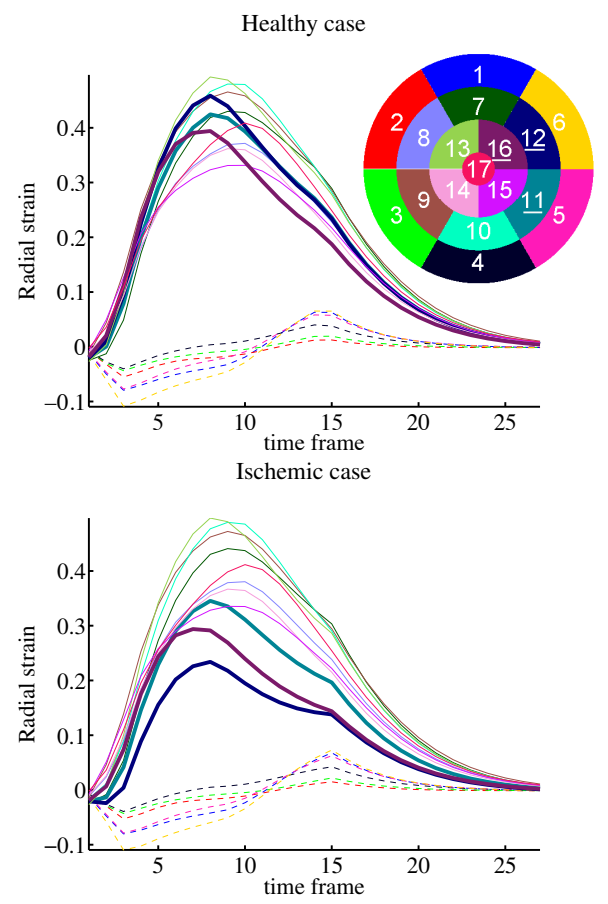

Fig. 4. Radial strain curves for the healthy and ischemic simulations. See the Bull's eye diagram for color legend. Dashed curves indicate basal segments. Non-basal ischemic segments are plotted in bold and underlined in the Bull's eye diagram.

where $a_{M A X}$ is the maximum $a$ value, $K=70$ is a constant and the power operation compensates the log compression traditionally performed in the ultrasound device prior to display. The normalized scatter map is set as the input of a fast ultrasound simulation [8] that computes radio-frequency signals on each scan line, and further performs envelope detection and scan conversion.

\section{RESULTS}

We present two synthetic sequences obtained with the proposed framework corresponding to one healthy and one ischemic motion pattern. A standard 3D US sequence was acquired from a healthy volunteer using a iE33 (Philips Medical Systems, Bothell WA, USA) echocardiography system. The first frame of the image sequence was considered to be the end of diastole while the end of systole was visually determined based on the aortic valve closure.

The synthetic motion was obtained from the mechanical simulation. For the ischemic simulation, peak contraction and stiffness values were modified in the territory of the left circumflex artery. To mimic the rigidity of the mitral annulus, basal segments were associated to high stiffness values. The $\mathrm{E} / \mathrm{M}$ model provides the benchmark strain curves that can be used to evaluate $3 \mathrm{D}$ speckle tracking algorithms. The resulting radial strain curves obtained for the two cases are plotted in Fig. 4. Basal strain curves (in dashed) have lower values in both the ischemic and the healthy cases. Ischemic regions 


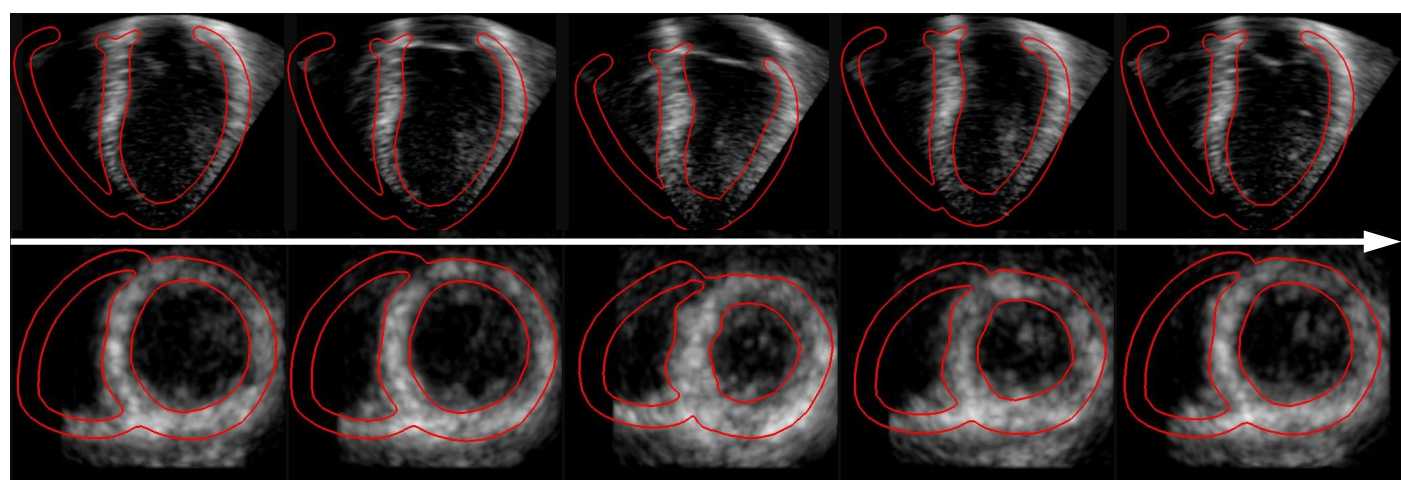

Fig. 5. Simulated sequence (top row: long axis view and bottom row: short axis view) corresponding to a healthy case. The 'benchmark' E/M mesh is superimposed over the simulated image. A video showing the entire simulated sequence is available at http://bit. ly/sim_us. An animation comparing ischemic and healthy simulations can be downloaded from http://bit.ly/sim_us2.

showed reduced radial strain values, they are underlined in the Bull's eye and plotted in bold. Taking as input the image sequence and the sequence of $\mathrm{E} / \mathrm{M}$ meshes, a scatter map was generated as described in Sect. 2.3. After simulation of the ultrasound acquisition [8], the resulting synthetic image sequence is plotted in Fig. 5, with the E/M meshes overlaid on the image and two links to visualize the sequences of images and meshes. Generated images and meshes are also attached to this submission. The realism of this simulated data has to be compared with state of the art strategies, as plotted in Fig. 1. The inclusion of a real clinical recording in the pipeline adds realistic challenges: presence of pericardium, valves, etc. The speckle texture inside the myocardium is realistic and can be adapted to several probe designs [8]. Motion within the myocardium is unbiased to any image registration algorithm. Note that despite the fact that two cases have been illustrated here, several intermediate cases can be generated to study the sensitivity of any image-based strain quantification technique under a controlled and reproducible environment.

\section{CONCLUSION}

We proposed a novel framework for the generation of highly realistic 3D ultrasound images. The realism of the proposed data represents an important shift with respect to state of the art strategies. First exemplary datasets are available for download. They include all generated meshes, providing ground truth landmarks for validating tracking algorithms, and synthetic images. The generated sequences and meshes can be downloaded from http://bit. ly/synthusdata.

As future work, we will extend the database to span over a range of location and contraction values. Other pathological shapes (hypertrophic, dilated) will be included by selecting representative 3D ultrasound sequences. This extended data will serve as basis for the comparative and quantitative evaluation of different strain quantification algorithms.

\section{REFERENCES}

[1] R. Jasaityte, B. Heyde, and J. D'hooge, "Current state of threedimensional myocardial strain estimation using echocardiography," J Am Soc Echocardiogr, vol. 26, no. 1, pp. 15 - 28, 2013.

[2] Q. Duan, P. Moireau, E. D. Angelini, D. Chapelle, and A. F. Laine, "Simulation of 3D US with a realistic electro-mechanical model of the heart," in Proc. of FIMH, 2007, pp. 463-473.

[3] C. Butakoff, S. Balocco, S. Ordas, and A. Frangi, "Simulated 3D US LV cardiac images for active shape model training," in Proc. of SPIE, 2007, vol. 6512, pp. 65123U-8.

[4] M. De Craene, S. Marchesseau, B. Heyde, H. Gao, M. Alessandrini, et al., "3D strain assessment in US (Straus): A synthetic comparison of five tracking methodologies," IEEE TMI, vol. 32, no. 9, pp. 1632-1646, 2013.

[5] M. Alessandrini, H. Liebgott, D. Friboulet, and O. Bernard, "Simulation of realistic echocardiographic sequences for ground-truth validation of motion estimation," in Proc. of IEEE ICIP, 2012, pp. 2329-2332.

[6] A. Prakosa, M. Sermesant, H. Delingette, S. Marchesseau, E. Saloux, et al., "Generation of synthetic but visually realistic time series of cardiac images combining a biophysical model and clinical images," IEEE TMI, vol. 32, no. 1, pp. 99-109, 2013.

[7] S. Marchesseau, H. Delingette, M. Sermesant, M. Sorine, K. Rhode, et al., "Preliminary specificity study of the BestelClement-Sorine electromechanical model of the heart using parameter calibration from medical images," J Mech Behav Biomed Mater., vol. 20, pp. 259-271, 2013.

[8] H. Gao, H. F. Choi, P. Claus, S. Boonen, S. Jaecques, et al., "A fast convolution-based methodology to simulate 2-D/3-D cardiac US images," IEEE TUFFC, vol. 56, no. 2, pp. 404-409, 2009.

[9] O. Ecabert, J. Peters, H. Schramm, C. Lorenz, J. Von Berg, et al., "Automatic model-based segmentation of the heart in CT images," IEEE TMI, vol. 27, no. 9, pp. 1189-1201, 2008.

[10] L. D. Jacobs, I. S. Salgo, S. Goonewardena, L. Weinert, P. Coon, D. Bardo, O. Gerard, P. Allain, et al., "Rapid online quantification of left ventricular volume from real-time threedimensional echocardiographic data," European Heart Journal, vol. 27, no. 4, pp. 460-468, 2006. 